\title{
Use of CAT scanning in the diagnosis and management of hepatic artery aneurysm
}

\author{
C C KIBBLER, D L COHEN, J K CRUICSHANK, S S KUSHWAHA, \\ MARSHA Y MORGAN, AND R D DICK \\ From the Departments of Medicine and Radiology, The Royal Free Hospital, London and the \\ Department of Medicine, Central Middlesex Hospital, London
}

SUMmaRY Hepatic artery aneurysms occur infrequently and are often difficult to diagnose. Non-invasive procedures such as ultrasound or computer assisted tomography have not been considered suitable for definitive diagnosis and angiography is usually required. A patient is described in whom a mycotic hepatic artery aneurysm developed during the course of subacute bacterial endocarditis. The definitive diagnosis was made using computer assisted tomography with intravenous contrast enhancement and angiography was undertaken only as a prelude to transcatheter embolisation. The same technique was used to monitor progress after embolisation precluding the need for follow up angiography. As $80 \%$ of patients with hepatic artery aneurysm present for the first time after aneurysm rupture, the mortality associated with this condition is high. More widespread use of intravenous contrast enhanced tomography for abdominal examination in patients with unexplained abdominal pain might result in earlier diagnosis of this condition.

Hepatic artery aneurysms occur infrequently and are often difficult to diagnose. In many patients the diagnosis is only made after aneurysm rupture so that only one patient in five with this condition survives even today. Until now angiography has been considered essential for the diagnosis; accurate, non-invasive methods of investigation might allow for earlier diagnosis and intervention. We describe a patient in whom the diagnosis of mycotic aneurysm of the the hepatic artery was made using computer assisted tomography (CAT) with intravenous contrast enhancement obviating the need for diagnostic angiography. The same procedure was used for monitoring the lesion after transcatheter embolisation.

\section{Case report}

A Pakistani boy emigrated to Great Britain in 1964 at the age of 13 . He was in good health and worked full time as a chef until January 1983 when gradually over a few days he developed pains in his knees, ankles, and wrists. Over the next three months he

Address for correspondence: Dr M Y Morgan. Medical Unit, Royal Free Hospital. Pond Street. Hampstead. London NW3 2QG.

Received for publication 21 August 1984. was treated with a variety of non-steroidal antiinflammatory agents but with little effect. During this time his general condition deteriorated, he lost $12.7 \mathrm{~kg}$ in weight and developed right upper quadrant pain. In May 1983 he was admitted to hospital for further investigation.

On examination he was cachectic, his temperature was $38^{\circ} \mathrm{C}$ and several joints were painful and swollen. He had a collapsing pulse and an early diastolic decrescendo murmur at the left sternal edge together with a diastolic murmur in the mitral area: there were no signs of cardiac failure and the rest of the examination was unremarkable.

Initial investigations showed the following: haemoglobin $8.3 \mathrm{~g} / \mathrm{dl}$ (reference range 13.0-17.5 $\mathrm{g} / \mathrm{dl})$, white cell count $10400 \times 10^{9} / 1(4-11,000 \times$ $\left.10^{9} / \mathrm{l}\right)$, ESR $125 \mathrm{~mm} / \mathrm{h}(<17 \mathrm{~mm} / \mathrm{h})$, serum bilirubin $4 \mu \mathrm{mol} / 1 \quad(0.2 \mathrm{mg} / 100 \mathrm{ml})[5-17 \mu \mathrm{mol} ; 0 \cdot 3-1 \cdot 0$ $\mathrm{mg} / 100 \mathrm{ml}$, aspartate transaminase $74 \mathrm{U} / \mathrm{l}(5-40$ $\mathrm{U} / \mathrm{l})$, alkaline phosphatase $840 \mathrm{U} / \mathrm{l}(118 \mathrm{KAu} / 100$ $\mathrm{ml}$ ) [30-110 U/l; 3-13 KAu/100 ml]. The urea and electrolytes were normal but there was persistent microscopic haematuria. An echocardiogram revealed aortic regurgitation with vegetations on the aortic valve, but no evidence of mitral valve disease. Streptococcus mutans was grown in blood culture.

He was treated with intravenous benzyl penicillin 
$8 \mathrm{~g}$ daily in divided doses for six weeks together with intravenous gentamycin $240 \mathrm{mg}$ daily in divided doses for the first two weeks. Back titrations of serum on this regime showed a predose bacteriocidal level at dilutions of $1: 128$ or more.

Over the next two weeks his general condition began to improve. However, his right upper quadrant pain persisted, his liver edge became palpable 3 $\mathrm{cm}$ below the right costal margin and serum alkaline phosphatase values rose further. An abdominal ultrasound carried out at this time showed a $2 \times 3 \mathrm{~cm}$ cystic mass in relation to the right portal vein with features suggestive of an hepatic artery aneurysm. A CAT scan with intravenous contrast enhancement confirmed the presence of a $3 \mathrm{~cm}$ diameter intrahepatic aneurysm in relation to the right hepatic artery (Fig. 1). The aneurysm was considered suitable for transcatheter embolisation. Preembolisation selective hepatic artery angiography positioned the aneurysm on one of the intrahepatic branches of the right hepatic artery $2.5 \mathrm{~cm}$ lateral to its major bifurcation, although there was a small feeding tributary from the left hepatic artery (Figs. $2 a, b)$. The aneurysm was embolised using a mixture
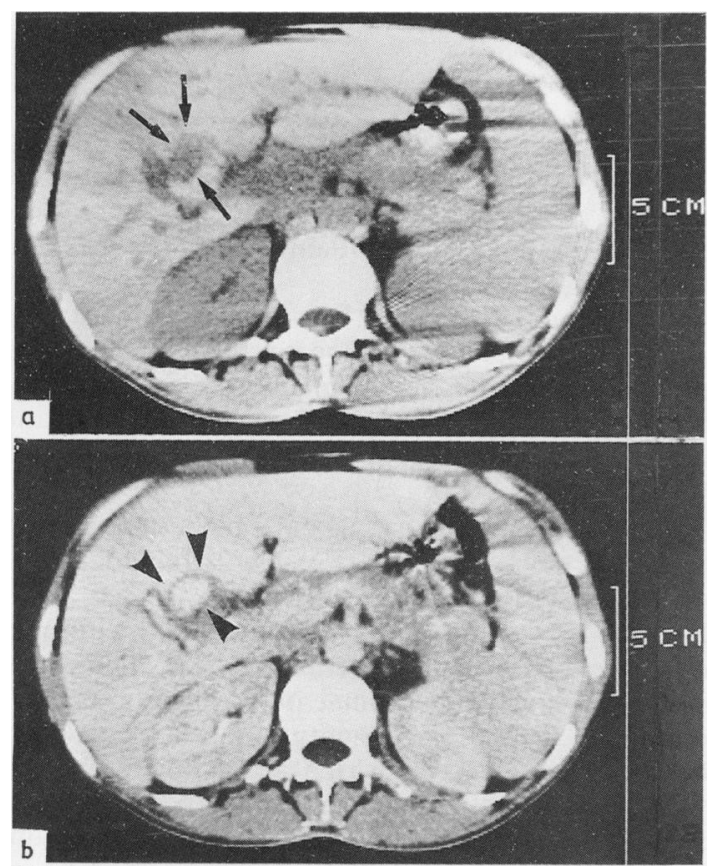

Fig. 1a, 1b CAT scans of the upper abdomen before and after contrast enhancement. The aneurysm shows as a filling defect (black arrows) which highlights after contrast injection (arrow heads). of $50 \%$ dextrose, absolute alcohol and sterispon particles. An immediate post embolisation angiogram showed successful obliteration of the aneurysm and its supplying blood vessels (Fig. 3).

The patient tolerated the procedure extremely well with no undue discomfort and with no significant change in liver function tests. The serum alkaline phosphatase values fell to normal over the next two weeks (Fig. 4) and a repeat CAT scan with intravenous contrast enhancement showed no evidence of recurrence of the lesion.

Four weeks after embolisation he underwent a successful aortic valve replacement with a StarrEdwards prosthesis. The aortic valve was tricuspid with thin redundant cusps which prolapsed; the presence of hard nodules in the ring of the noncoronary cusp suggested previous rheumatic damage. Long term treatment with warfarin was started.

He remains well eight months after aneurysmic embolisation and valve replacement. He has gained $9 \mathrm{~kg}$ in weight and has returned to gainful, full time employment. A further CAT scan with intravenous contrast enhancement showed no evidence of recurrence of the lesion (Fig. 5).

\section{Discussion}

Aneurysm of the hepatic artery was first described by Wilson in 1809 as a post mortem finding in a 50 year old man who had exsanguinated following its rupture. ${ }^{12}$ The condition is still rarely diagnosed in life and carries a high mortality. In a review of the literature in 1966, Guida and Moore ${ }^{1}$ reported 170 cases of whom only 23 had been successfully treated and survived. By 1975 , approximately 300 cases had been reported but only $60(20 \%)$ had been successfully treated. ${ }^{3-5}$ In the last decade a small number of further cases have been reported usually to illustrate newer techniques of management such as transcatheter embolisation. ${ }^{6-8}$

Before the advent of antibiotics the majority of hepatic artery aneurysms were mycotic in origin and were almost invariably associated with endocarditis. ${ }^{9}$ In recent years mycotic aneurysms have accounted for only $10 \%$ of all hepatic artery aneurysms. ${ }^{10}$ The percentage of aneurysms arising in association with infection is, however, likely to become more frequent with increasing intravenous drug abuse, ${ }^{7}$ prosthetic valve replacement, prolonged intravenous therapy and with an increasing number of immunocompromised individuals. Trauma is implicated in the development of approximately $20 \%$ of hepatic artery aneurysms. This includes blunt abdominal trauma, penetrating injury such as knife or gunshot wounds or inadvertent surgical trauma. ${ }^{10-12}$ Arteriosclerotic changes are 

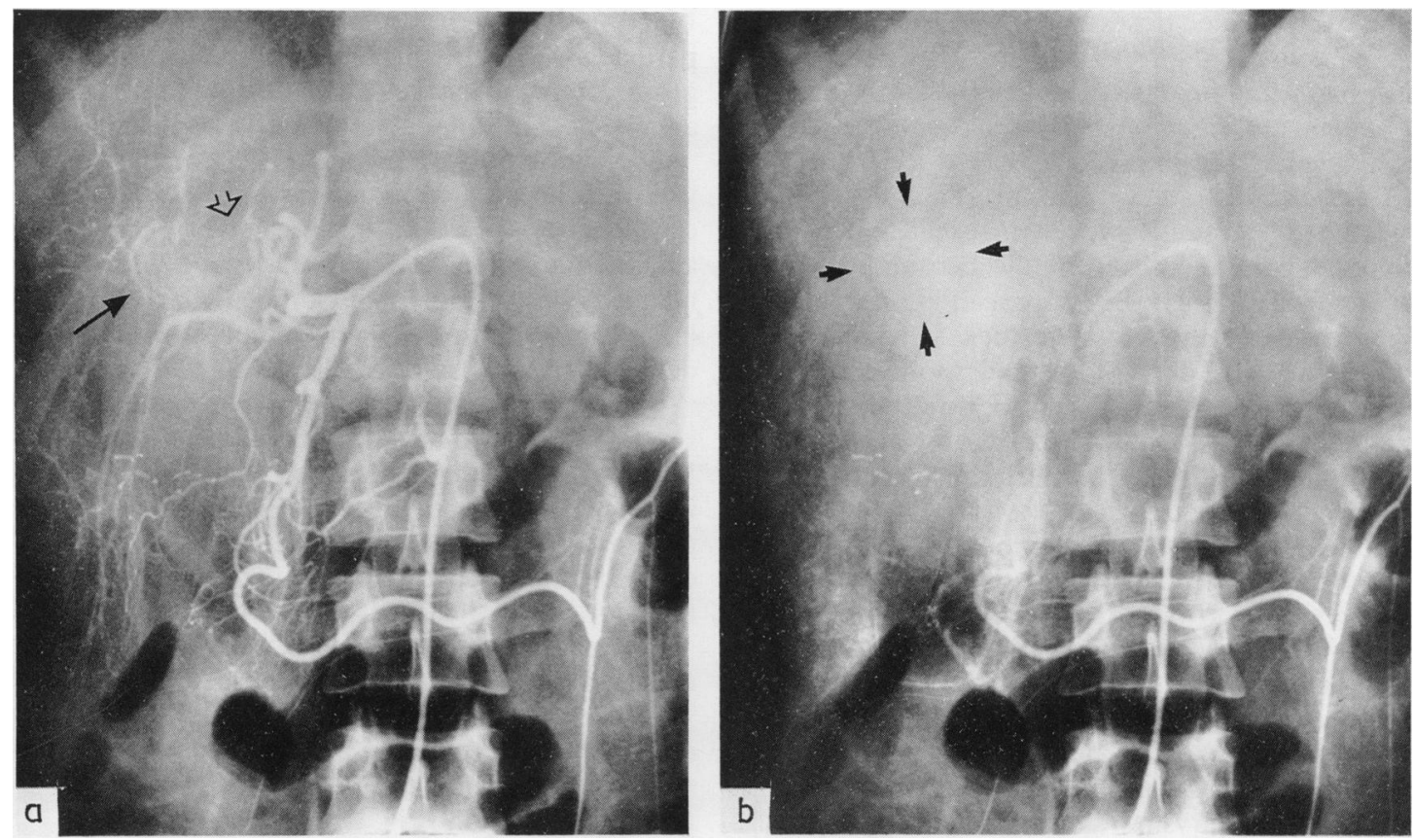

Fig. 2a Coeliac arteriogram showing a $3 \mathrm{~cm}$ false aneurysm of one of the intrahepatic branches of the right hepatic artery $2.5 \mathrm{~cm}$ lateral to its major bifurcation (black arrow). There is a small feeding vessel from the left hepatic artery (open arrow). $2 \mathrm{~b}$ Late phase of coeliac angiogram showing filling of the aneurysm (black arrows)

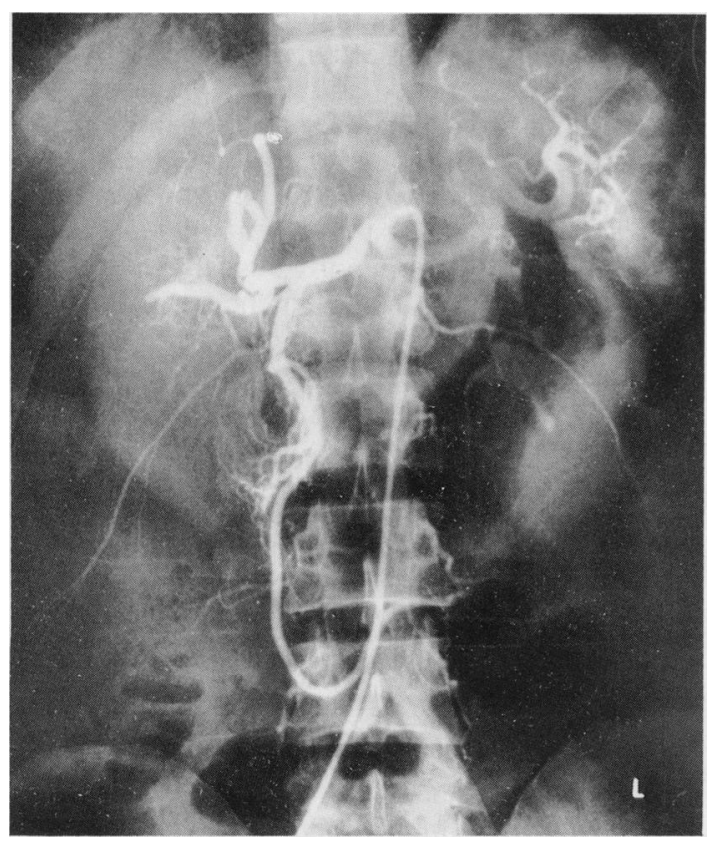

Fig. 3 Coeliac angiogram immediately post-embolisation showing obliteration of the aneurysm and its feeding vessels. important in the aetiology of between one-third to one-half of hepatic artery aneurysms while less frequent causes include polyarteritis nodosa, ${ }^{13}$ Marfan's syndrome ${ }^{14}$ and inflammation of the gall bladder and pancreas.

Approximately $80 \%$ of hepatic artery aneurysms are extrahepatic, in which location they may be true or false in nature. The remaining $20 \%$ are intrahepatic, occur more frequently in the right hepatic artery than the left, and are usually false aneurysms. ${ }^{1} 101516$

Hepatic artery aneurysms have been described in patients from 10 to 83 years of age ${ }^{1}$ with a mean age of 40; the lesion appears commoner in men. ${ }^{1} 10^{15}$ Symptoms and signs are extremely varied with only one third of patients presenting with Quincke's classical triad of abdominal pain, haemobilia and obstructive jaundice. ${ }^{17}$ Eighty per cent of patients complain of epigastric or right upper quadrant pain which occasionally radiates to the back or right shoulder. ${ }^{12}{ }^{17}$ Haemobilia occurs in $62 \%$ and jaundice in just over one-half. ${ }^{5}$ In as many as $80 \%$ of patients the aneurysm has ruptured by the time of presentation ${ }^{17}$ either into the peritoneal cavity or into the biliary tree with equal frequency. ${ }^{14}$ The commonest symptoms after aneurysm rupture are 
Fig. 4 Changes in serum bilirubin, serum aspartate transaminase (AST) and serum alkaline phosphatase (ALP) immediately before and after embolisation of an hepatic artery aneurysm.

$$
\begin{aligned}
& \text {-reference range } \\
& \text { bilirubin: } \mu \text { mol/l x } 0.0585= \\
& \mathrm{mg} / 100 \mathrm{ml} \text {; alkaline } \\
& \text { phosphatase: U/l } \times 0.141= \\
& \text { KAul100 ml. }
\end{aligned}
$$

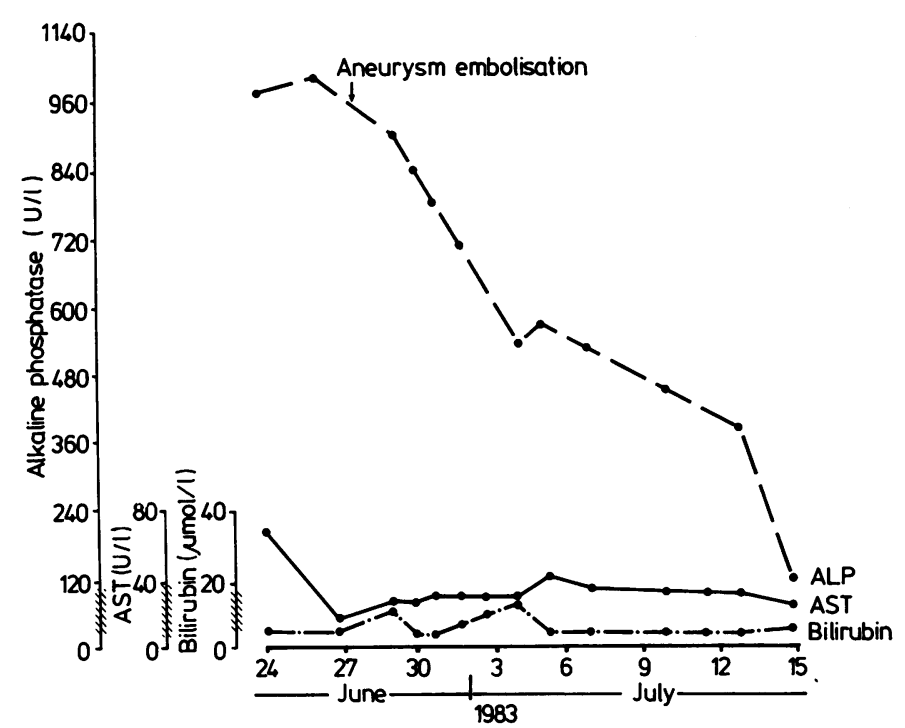

abdominal pain and haemorrhage. ${ }^{17}$ On physical examination there may be an upper abdominal mass or an arterial bruit though both are uncommon; $;^{12} 1718$ hepatomegaly as observed in our patient is rare.

The diagnosis of hepatic artery aneurysm is difficult to make. Routine investigations are usually unhelpful; there may be a leucocytosis and serum bilirubin, aspartate transaminase and alkaline phosphate values may be raised. Plain films of the abdomen may occasionally show a calcified rim in the upper abdomen. ${ }^{2}$ Similarly, contrast studies of the upper gastrointestinal tract may show displace-

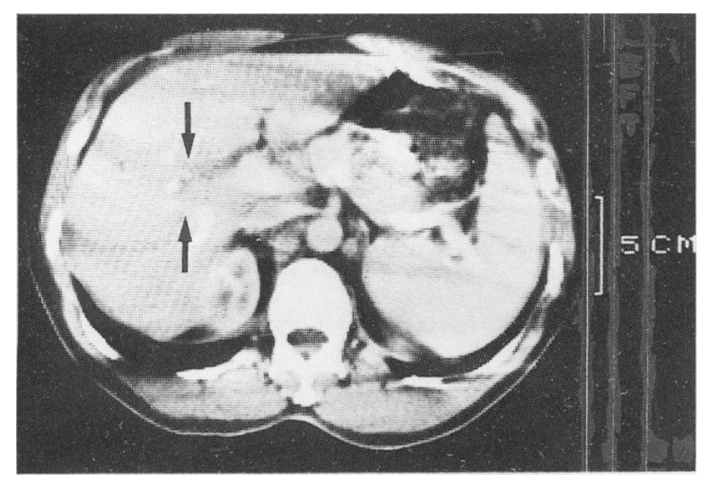

Fig. 5 CAT scan with enhancement eight months post hepatic artery aneurysm embolisation. The aneurysm shows as a filling defect (black arrows) but does not fill with contrast. ment of the duodenal curve by an extrinsic mass. ${ }^{12}$ In reports to date ultrasound and CAT scanning have shown a mass but have been of little help in determining its origin. ${ }^{19}$ In one report ${ }^{19}$ CAT scan and ultrasound showed a large epigastric mass of mixed cystic and solid components which on angiography proved to be a large aneurysms of the common hepatic artery. The use of intravenous contrast enhancement would have showed the vascular nature of this mass and allowed more definitive CAT scan diagnosis. In our patient use of CAT scanning with intravenous contrast enhancement enabled the diagnosis to be made without resort to more invasive procedures. Angiography was carried out subsequently to delineate the feeding vessels of the aneurysm as a preliminary to transcatheter embolisation. Angiography was not, however, needed for definitive diagnosis.

Once the diagnosis of hepatic artery aneurysm has been made, aggressive treatment is necessary. A number of surgical approaches are available but the most successful procedure is simple ligation or ligation and excision of the aneurysm. ${ }^{13} 510-$ 1315171820 In the absence of a collateral circulation, efforts should be made to repair the vessel involved in order to prevent massive liver necrosis $^{13} 5^{10-13} 15171819$ though recent evidence suggests that even in the absence of a demonstrable collateral circulation, ligation may still be the preferred procedure. ${ }^{20-22}$ In the last decade transcatheter embolisation has been used in selective patients as an effective alternative for the management of intrahepatic aneurysms. ${ }^{6-8}$ This procedure 
is generally well tolerated as in our patient.

After the definitive procedure it is customary to perform repeat angiography during the follow-up period. In our patient, however, CAT scanning with intravenous contrast enhancement was used as the monitoring procedure.

Successful management of hepatic artery aneurysm depends on early diagnosis and intervention. The diagnosis should always be suspected in patients with a recent or remote history of septicaemia or endocarditis, with a history of recent or remote abdominal trauma including surgery or otherwise unexplained upper abdominal pain. Examination of the upper abdomen with CAT scanning with intravenous contrast enhancement in such patients may enable the diagnosis of hepatic artery aneurysm to be made without resort to more invasive diagnostic procedures.

We thank Dr V L Steinberg, Dr E Jepson, and Professor Dame Sheila Sherlock for allowing us to report this patient who was under their care.

MYM is William Gibson Scholar of the Royal Society of Medicine, London.

\section{References}

1 Guida PM, Moore SW. Aneurysm of the hepatic artery: report of five cases with a brief review of the previously reported cases. Surgery 1966; 60: 299-310.

2 Payne JE, Kemmerer WT. Hepatic artery aneurysm with rupture into general peritoneal cavity. $J$ Trauma 1967; 7: 793-7.

3 Rutten APM, Sikkenh PJH. Aneurysm of the hepatic artery: reconstruction with saphenous vein graft. $\mathrm{Br} J$ Surg 1971; 50: 262-6.

4 Ariyan S, Cahow CE, Greene FL, Stansel HC Jr. Successful treatment of hepatic artery aneurysm with erosion into the common duct. Ann Surg 1975; 182: 169-72.

5 Croom RD III, Frantz PT, Thomas CG, Hothem AO.
Anerysms of the hepatic artery. South Med J 1976; 69: 1013-7.

6 Goldblatt M, Goldin AR, Shaff MI. Percutaneous embolisation for the management of hepatic artery aneurysms. Gastroenterology 1977; 73: 1142-6.

7 Porter LLIII, Houston MC, Kadir S. Mycotic anerysms of the hepatic artery: treatment with arterial embolisation. Am J Med 1979; 67: 697-701.

8 Kadir S, Athanasoulis CA, Ring EJ, Greenfield A. Transcatheter embolisation of intrahepatic artery aneurysms. Radiology 1980; 134: 335-9.

9 Stengal A, Wolferth CC. Myotic aneurysms of intravascular origin. Arch Intern Med 1923; 31: 527-54.

10 Rutherford RB. Vascular surgery. Philadelphia: WB Saunders, 1977: 676-8.

11 Bussuttil RW, Brin BJ. The diagnosis and management of visceral artery aneurysms. Surgery 1980; 88: 619-24.

12 Hubens A, DeShepper A. Hepatic artery aneurysm: a pitfall in biliary surgery. Br J Surg 1979; 66: 259-61.

13 Stanley JC, Thompson NW, Fry WJ. Splanchnic artery aneurysms. Arch Surg 1970; 101: 689-97.

14 Santiago-Delphin EA, Marquez E, Rodriquez OL, Oliveras FE, Baldizon C, Martinez-Cabruja R. Perforated hepatic artery aneurysm and multiple aneurysms in incomplete Marfan syndrome. Ann Surg 1972; 176: $772-6$.

15 Haimovici H. Vascular surgery - principles and techniques. New York: McGraw-Hill, 1976: 383-4.

16 Alexander RA. Lawrence GH. Operative repair of hepatic artery aneurysm. Arch Surg 1966; 93: 409-14.

17 Harlafris NN, Akin JG. Hemobilia from ruptured hepatic artery aneurysm. Ann J Surg 1977; 133: 229-32.

18 Abraham RA, Stone J, Conn JH, Chavez CM. Hepatic artery aneurysm - diagnosis and successful treatment. Angiology 1971; 22: 134-40.

19 Countryman D, Norwood S, Register D, Torma M, Andrassy R. Hepatic artery aneurysm: report of an unusual case and review of the literature. Am Surg 1983; 49: 51-4.

20 Kirklin JW, Shocket E, Comfort MW, Huizenga KA. Treatment of hepatic artery aneurysm by excision. Report of case. Ann Surg 1955; 142: 110-4.

21 Asbury GF. Ruptured hepatic artery aneurysm. Ann Surg 1970; 36: 631-4.

22 Erskine JM. Ruptured intrahepatic aneurysm. Arch Surg 1973; 106: 219-22. 